\title{
Voltage Sag Source Location Estimation Based on Optimized Configuration of Monitoring Points
}

\author{
Ganyun LV, Chenjie CHU, Yu ZANG, and Guangyu CHEN
}

\begin{abstract}
In order to reduce the influence of monitoring blind area caused by the uncertainty of fault resistance on voltage sag source location in transmission line short-circuit fault, a voltage sag source location estimation method based on optimal configuration of system monitoring points and fault parameter estimation is proposed. Considering the observability of voltage sag in various short-circuit faults, the importance index of measuring points in the system is proposed, so as to construct the optimal configuration model of monitoring points. Based on the optimal configuration scheme of monitoring points, the sag source location model is established, and the adaptive cuckoo algorithm is used to estimate the parameters of fault line, fault resistance and fault distance. Finally, the simulation is verified by IEEE-30 node standard test system. The results show that the proposed method has higher fault location accuracy and more engineering applicability than the traditional method.
\end{abstract}

Index Terms - Cuckoo search, fault location, fault resistance, optimal configuration of monitoring points, sag source location model, voltage sag.

\section{INTRODUCTION}

A $\mathrm{S}$ one of the most important indicators of power quality, voltage sag has caused a series of serious problems to a large number of power systems and sensitive power supply and consumption equipment of users in recent years [1]-[3], including tripping of computers and electronic equipment, abnormal operation of lighting equipment, motors, wind power generation, speed regulation system and a variety of industrial equipment. According to the statistics of the American Academy of Electrical Sciences, the economic loss of the United States due to voltage sag is more than 20 billion US dollars every year [4]. China's annual economic loss due to power quality problems reaches 1.5 trillion yuan [5]. Nowadays, in addition to traditional power generation sources such as hydropower, gas turbine and fossil fuel power generation, many new power generation sources have been added to the modern power system, such as photovoltaic and wind power genera-

Manuscript received July 1, 2021; revised August 9, 2021; accepted August 31, 2021. Date of publication September 30, 2021; date of current version September 22, 2021. This work was supported in part by the National Natural Science Foundation of China under Grant 51577086 and in part by Major University Science Research Project of Jiangsu Province under Grant 19KJA 510012 and in part by Jiangsu Six Talent Peaks Project under Grant TD-XNY004.

All authors are with the Electrical Engineering Department, Nanjing Institute of Technology, Nanjing 211167, China (e-mail: ganyun_lv@ njit.edu.cn).

Digital Object Identifier 10.24295/CPSSTPEA.2021.00023 tion, which increases the complexity of the power system [6], and poses a certain challenge for voltage sag fault diagnosis [7]. Accurate positioning of voltage sag source will help power grid companies eliminate faults as soon as possible and clarify the responsibilities of power suppliers and users; On the other hand, it can better coordinate the disputes between power departments and users in the power market environment, and improve the operation, maintenance and management efficiency of power grid.

Therefore, it is of great practical significance to establish a perfect and reliable voltage sag monitoring and positioning system. The key to solve the above problems is to optimize the configuration of limited power quality monitors to realize the voltage monitoring of sensitive load nodes in the whole network, and locate the voltage sag source on this basis. There are many methods for optimal configuration of voltage sag monitoring points. The proposed methods mainly include monitor reach area (MRA) [8]-[9] and fault location (FL) [10]. MRA method aims at minimizing the number of monitoring devices and configuring monitoring points according to the given voltage sag threshold, which has some problems, such as difficult threshold selection and deletion of non monitoring point information. FL method configures monitoring points by taking the observable disturbance sources of the whole network as the target through the fault location matrix. There are many fault points obtained by this method. Moreover, the fault resistance is ignored in the modeling of the above methods. Due to the objective existence of fault resistance in case of short-circuit fault in power system, the engineering applicability will be reduced. Literature [11] considers the existence of fault resistance and proposes an optimal configuration scheme of monitoring points based on voltage sag estimation and fault location. However, this method ignores that in practical engineering applications, the monitoring device only records sag events whose amplitude is lower than the threshold.

Scholars have carried out a lot of research in the field of voltage sag source location. Literatures [12], [13] proposed a transient disturbance location method based on the perturbation power and energy method to determine which side of the voltage sag source comes from the monitoring equipment. Literatures [14]-[18] proposed a positioning method based on the real part of the impedance, including the polarity of the real part of the equivalent impedance, the slope of the system trajectory, the polarity of the real part of the current, and the positioning method based on the distance impedance relay. Make sure 
that the source of interference is upstream or downstream of the monitoring device. Literature [19] proposes a voltage sag source location method based on semi supervised support vector machine. Combined with multiple location feature quantities, the upstream and downstream classification surface is constructed by using the idea of two classification to realize voltage sag source location. The accuracy of this location method is higher than that of single feature criterion. The above method is to distinguish the relative orientation of the interference source causing voltage sag based on the monitoring information of a single measuring point, which is easy to locate errors when the measurement accuracy is not high.

With the gradual improvement of power quality monitoring system, scholars at home and abroad have also carried out a lot of research on sag source location method based on multi monitoring point sag information. Literatures [20], [21] match the measured sag data with the samples in the voltage sag database, and locate the fault point based on the fault distance distribution function. Its accuracy is greatly affected by the database samples and the accuracy of the data measurement. Literature [22] considers the voltage amplitude and phase at the same time, and uses the real and imaginary part separation method for the positioning equation to determine the fault location and fault resistance. This method is too computationally expensive and can only be effective if the interference source type is accurately identified. Literature [23] combines the observable area of the measuring point and the sag source location to determine the possible faulty line, then estimates the line fault type, and finally assumes the virtual fault point, and uses the error analysis based on the fault distance distribution function combined with the actual measured value of the monitoring point to locate the location of the voltage sag source, but the positioning accuracy is affected by the fault resistance. Literature [24] proposed a method for locating voltage sag sources based on the optimal configuration of monitoring points. The least squares estimation model was established by measuring voltage amplitude and fault distance as state variables, but did not consider the impact of fault resistance on location. Literature [25] proposed a voltage sag source location method based on the positive sequence voltage correlation of multiple measuring points and typical pattern matching, which effectively overcomes the influence of fault type and transition resistance on the location of the sag source, but it needs to be arranged in the system more monitoring points.

The sag depth of the measuring point is related to the three variables of fault location, fault type, and fault resistance. When any factor changes, the voltage of the measuring point will also change [25]. The existing voltage sag monitoring point configuration methods generally ignore the parameter of fault resistance in modeling, which reduces its engineering applicability. In practical engineering application, the monitoring device will only record the voltage sag event whose amplitude is lower than the set threshold, but will not record the signal all the time.

This paper presents a new configuration scheme of voltage

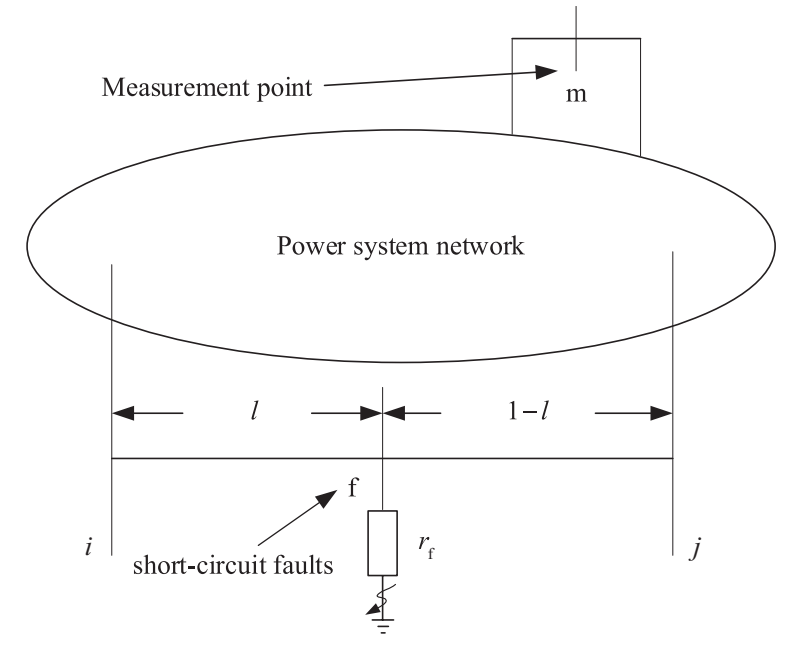

Fig. 1. Schematic diagram of system short-circuit failure.

sag monitoring points. Firstly, the critical fault resistance matrix is constructed based on power grid parameters and short-circuit calculation, and the importance matrix of measuring points is constructed based on the average voltage sag level of the whole network after fault, and then the optimal configuration scheme of monitoring points is determined. On this basis, taking the fault line, fault resistance and fault distance as optimization variables and measuring point voltage as measurement, the sag source location state estimation model is constructed. The model solves the mixed integer nonlinear programming (MINLP) problem, solved by adaptive cuckoo algorithm in this paper. The simulation results of IEEE-30 bus test system show that the method in this paper is correct and effective, and has more engineering applicability than the traditional method.

\section{Calculation Model of Fault Voltage Amplitude}

The schematic diagram of system short-circuit fault is shown in Fig. 1. Assuming that it is a short-circuit fault point on the line, is the normalized distance from the fault point to the head end node of the line, and is a monitoring point in the system, the self-impedance at the fault point and the mutual impedance between each are obtained by the following equations.

$$
\begin{aligned}
Z_{\mathrm{ff}}^{c}= & (1-l)^{2} Z_{i i}^{c}+l^{2} Z_{j j}^{c} \\
& +2 l(1-l) Z_{i j}^{c}+l(1-l) Z_{i j}^{c} \\
Z_{\mathrm{mf}}^{c} & =(1-l) Z_{\mathrm{m} i}^{c}+l Z_{\mathrm{m} j}^{c}
\end{aligned}
$$

Where $Z_{i i}^{c}$ and $Z_{i i}^{c}$ are the self-impedances of the respective nodes; $Z_{\mathrm{m} i}^{c}, Z_{\mathrm{m} j}^{c}, Z_{i j}^{c}$ are the mutual impedances between the nodes; $Z_{i j}^{c}$ are the line impedance values; $c=0,1,2$ are the zero sequence, positive sequence, and negative sequence respectively, See reference [8] for detailed formulas. If it is assumed that the voltage of each node before the fault is 1 $\mathrm{pu}$, the fault resistance is represented by. When various faults $f$ occur at the fault points in Fig. 1, the voltage amplitudes of 
each node are shown in (3)-(6).

For three-phase grounding fault, they are

$$
V_{\mathrm{m}}=1-\frac{Z_{\mathrm{mf}}^{1}}{Z_{\mathrm{ff}}^{1}+r_{\mathrm{f}}}
$$

For phase to phase short-circuit fault of phase BC, they are

$$
\left\{\begin{array}{l}
V_{\mathrm{m}, \mathrm{A}}=1-\frac{Z_{\mathrm{mf}}^{1}-Z_{\mathrm{mf}}^{2}}{Z_{\mathrm{ff}}^{1}+Z_{\mathrm{ff}}^{2}+2 r_{\mathrm{f}}} \\
V_{\mathrm{m}, \mathrm{B}}=\alpha^{2}-\frac{\alpha^{2} Z_{\mathrm{mf}}^{1}-\alpha Z_{\mathrm{mf}}^{2}}{Z_{\mathrm{ff}}^{1}+Z_{\mathrm{ff}}^{2}+2 r_{\mathrm{f}}} \\
V_{\mathrm{m}, \mathrm{C}}=\alpha-\frac{\alpha Z_{\mathrm{mf}}^{1}-\alpha^{2} Z_{\mathrm{mf}}^{2}}{Z_{\mathrm{ff}}^{1}+Z_{\mathrm{ff}}^{2}+2 r_{\mathrm{f}}}
\end{array}\right.
$$

For two-phase grounding fault at phase B and C, they are

$$
\left\{\begin{array}{l}
V_{\mathrm{m}, \mathrm{A}}=1- \\
\frac{\left(Z_{\mathrm{ff}}^{2}+Z_{\mathrm{ff}}^{0}+2 r_{\mathrm{f}}\right) Z_{\mathrm{mf}}^{1}-\left(Z_{\mathrm{ff}}^{0}+r_{\mathrm{f}}\right) Z_{\mathrm{mf}}^{2}-\left(Z_{\mathrm{ff}}^{2}+r_{\mathrm{f}}\right) Z_{\mathrm{mf}}^{0}}{Z_{\mathrm{ff}}^{1} Z_{\mathrm{ff}}^{2}+Z_{\mathrm{ff}}^{1} Z_{\mathrm{ff}}^{0}+Z_{\mathrm{ff}}^{2} Z_{\mathrm{ff}}^{0}+2 r_{\mathrm{f}}\left(Z_{\mathrm{ff}}^{1}+Z_{\mathrm{ff}}^{2}+Z_{\mathrm{ff}}^{0}\right)+3 r_{\mathrm{f}}^{2}} \\
V_{\mathrm{m}, \mathrm{B}}=\alpha^{2}- \\
\frac{\left(Z_{\mathrm{ff}}^{2}+Z_{\mathrm{ff}}^{0}+2 r_{\mathrm{f}}\right) \alpha^{2} Z_{\mathrm{mf}}^{1}-\left(Z_{\mathrm{ff}}^{0}+r_{\mathrm{f}}\right) \alpha Z_{\mathrm{mf}}^{2}-\left(Z_{\mathrm{ff}}^{2}+r_{\mathrm{f}}\right) Z_{\mathrm{mf}}^{0}}{Z_{\mathrm{ff}}^{1} Z_{\mathrm{ff}}^{2}+Z_{\mathrm{ff}}^{1} Z_{\mathrm{ff}}^{0}+Z_{\mathrm{ff}}^{2} Z_{\mathrm{ff}}^{0}+2 r_{\mathrm{f}}\left(Z_{\mathrm{ff}}^{1}+Z_{\mathrm{ff}}^{2}+Z_{\mathrm{ff}}^{0}\right)+3 r_{\mathrm{f}}^{2}} \\
V_{\mathrm{m}, \mathrm{C}}=\alpha- \\
\frac{\left(Z_{\mathrm{ff}}^{2}+Z_{\mathrm{ff}}^{0}+2 r_{\mathrm{f}}\right) \alpha Z_{\mathrm{mf}}^{1}-\left(Z_{\mathrm{ff}}^{0}+r_{\mathrm{f}}\right) \alpha^{2} Z_{\mathrm{mf}}^{2}-\left(Z_{\mathrm{ff}}^{2}+r_{\mathrm{f}}\right) Z_{\mathrm{mf}}^{0}}{Z_{\mathrm{ff}}^{1} Z_{\mathrm{ff}}^{2}+Z_{\mathrm{ff}}^{1} Z_{\mathrm{ff}}^{0}+Z_{\mathrm{ff}}^{2} Z_{\mathrm{ff}}^{0}+2 r_{\mathrm{f}}\left(Z_{\mathrm{ff}}^{1}+Z_{\mathrm{ff}}^{2}+Z_{\mathrm{ff}}^{0}\right)+3 r_{\mathrm{f}}^{2}}
\end{array}\right.
$$

For single phase to ground fault at phase A, they are

$$
\left\{\begin{array}{l}
V_{\mathrm{m}, \mathrm{A}}=1-\frac{Z_{\mathrm{mf}}^{1}+Z_{\mathrm{mf}}^{2}+Z_{\mathrm{mf}}^{0}}{Z_{\mathrm{ff}}^{1}+Z_{\mathrm{ff}}^{2}+Z_{\mathrm{ff}}^{0}+3 r_{\mathrm{f}}} \\
V_{\mathrm{m}, \mathrm{B}}=\alpha^{2}-\frac{\alpha^{2} Z_{\mathrm{mf}}^{1}+\alpha Z_{\mathrm{mf}}^{2}+Z_{\mathrm{mf}}^{0}}{Z_{\mathrm{ff}}^{1}+Z_{\mathrm{ff}}^{2}+Z_{\mathrm{ff}}^{0}+3 r_{\mathrm{f}}} \\
V_{\mathrm{m}, \mathrm{C}}=\alpha-\frac{\alpha Z_{\mathrm{mf}}^{1}+\alpha^{2} Z_{\mathrm{mf}}^{2}+Z_{\mathrm{mf}}^{0}}{Z_{\mathrm{ff}}^{1}+Z_{\mathrm{ff}}^{2}+Z_{\mathrm{ff}}^{0}+3 r_{\mathrm{f}}}
\end{array}\right.
$$

\section{Optimal Configuration of Monitoring Points Considering Fault Resistance and Importance of MeAsuring PoInts}

\section{A. Configuration Principle}

Generally, monitoring devices will only record voltage sag events whose amplitude is lower than the set threshold due to limited storage space. The actual significance of the configuration of the monitoring device is that when a voltage sag occurs anywhere in the system, it is captured by at least $b$ monitoring points. Whether the monitoring device can capture the voltage sag is the key.

For a system with $n$ nodes, the decision vector of the monitor- ing device is

$$
X=\left[\begin{array}{llllll}
x_{1} & x_{2} & \ldots & x_{i} & \ldots & x_{n}
\end{array}\right]
$$

Where $i=1,2, \cdots, n . x_{i}$ is given as

$$
x_{i}=\left\{\begin{array}{l}
1 \text { When node is a monitoring point } \\
0 \text { When node is not a monitoring point }
\end{array}\right.
$$

Considering that with the increase of fault resistance, the MRA of monitoring points in the system will become smaller [26], while the traditional monitoring point configuration scheme does not consider the fault resistance parameters, which leads to the failure of the monitoring device to record the voltage sag event to a great extent. In view of the shortcomings of the traditional model, the key variable of fault resistance needs to be introduced into the optimal configuration of monitoring points.

\section{B. Critical Fault Resistance Matrix}

When the fault location and the voltage sag threshold $V_{\text {th }}$ are known, the corresponding critical fault resistance can be obtained according to the inverse solution of (3) to (6). For asymmetric short-circuit faults, the maximum value of the critical fault resistance is taken as the critical fault resistance value of the fault point.

Define the critical fault resistance matrix as follows,

$$
R_{\mathrm{cri}}^{t}=\left[\begin{array}{cccc}
r_{\mathrm{cri}, 11}^{t} & r_{\mathrm{cri}, 12}^{t} & \cdots & r_{\mathrm{cri}, 1 n}^{t} \\
r_{\mathrm{cri}, 21}^{t} & r_{\mathrm{cri}, 22}^{t} & \cdots & r_{\mathrm{cri}, 2 n}^{t} \\
\vdots & \vdots & \ddots & \vdots \\
r_{\mathrm{cri}, m 1}^{t} & r_{\mathrm{cri}, m 2}^{t} & \cdots & r_{\mathrm{cri}, m n}^{t}
\end{array}\right]
$$

Where $t$ is the type of failure; $n$ is the number of nodes in the entire network; $m$ is the number of failure points set for the entire network. $r_{\text {cri, } i j}^{t}$ indicates the critical fault resistance at the node $j$ when a t-type fault occurs at the fault point.

In addition, the critical failure resistance matrix based on the decision-making scheme is defined as follows,

$$
R_{D}^{t}=\left[\begin{array}{cccc}
r_{D, 11}^{t} & r_{D, 12}^{t} & \cdots & r_{D, 1 n}^{t} \\
r_{D, 21}^{t} & r_{D, 22}^{t} & \cdots & r_{D, 2 b}^{t} \\
\vdots & \vdots & \ddots & \vdots \\
r_{D, n 1}^{t} & r_{D, n 2}^{t} & \cdots & r_{D, n n}^{t}
\end{array}\right]
$$

where $r_{D, i j}^{t}=r_{\mathrm{cri}, i j}^{t} x_{i}$.

\section{Short-Circuit Fault Voltage Matrix}

Taking into account that apart from a zero fault resistance short circuit, no other factors will cause a greater depth of voltage sag. Therefore, when the fault resistance is zero, the voltage sag of the whole network caused by the short-circuit fault is the largest. And under an asymmetric fault, any phase 
voltage of the node is less than the critical voltage, which will cause the voltage of the node to sag. That is, the key to judging whether a node has a voltage sag depends on the phase with the lowest voltage amplitude at that node.

If considering each phase $p(p \in\{\mathrm{a}, \mathrm{b}, \mathrm{c}\})$ and each shortcircuit fault $t(t \in\{1,2,3,4\})$, define the short-circuit fault matrix as follows,

$$
F V_{t}^{p}=\left[\begin{array}{cccc}
f v_{11} & f v_{12} & \cdots & f v_{1 n} \\
f v_{21} & f v_{22} & \cdots & f v_{2 n} \\
\vdots & \vdots & \ddots & \vdots \\
f v_{n 1} & f v_{n 2} & \cdots & f v_{n n}
\end{array}\right], t=1,2,3,4 p=\mathrm{a}, \mathrm{b}, \mathrm{c}
$$

where $f v_{i j}$ is the phase voltage value of the node $j$ when a fault occurs at the node $i$.

\section{Optimal Configuration Model of Monitoring Points}

Obtainable voltage sag observability matrix based on critical fault resistance matrix $M^{t}(i, j)$

$$
M^{t}(i, j)= \begin{cases}1 & R_{\text {cri }}^{t}>0 \\ 0 & \text { other }\end{cases}
$$

Then consider the considerable constraint conditions for the voltage sag of the whole network as

$$
\sum_{i=1}^{n} x(i) M^{t}(i, j) \geqslant b_{j}
$$

Where $b_{j}$ is the least the number of times that each fault point has been observed.

Due to the large uncertainty of the fault resistance, when the traditional monitoring point configuration scheme is adopted, the monitoring of the voltage sag may be omitted, and the observable rate of the voltage sag is defined as follows,

observable rate $=\frac{\text { number of measured sags }}{\text { total number of voltage sags caused by faults }}$

If $f\left(r_{i}^{t}\right)$ is used to represent the probability density function of the fault resistance when a t-type of short-circuit fault occurs at the fault point $i ; A_{\mathrm{cri}, i}^{t}$ and $A_{D, i}^{t}$ respectively represent the maximum value of the element in the row $i$ of the matrix $R_{\text {cri }}^{t}$ and $R_{D}^{t}$, and $r_{\min }^{t}$ represents the minimum value of the fault resistance in the t-type fault. Then the observable rate of voltage sag for the t-type fault at the fault point $i$ can be expressed as,

$$
P_{X, i}^{t}=\frac{\int_{r_{\min }^{t}}^{A_{D, i}^{t}} f\left(r_{i}^{t}\right) d r_{i}^{t}}{\int_{r_{\min }^{t}}^{A_{\mathrm{cr}, i}} f\left(r_{i}^{t}\right) d r_{i}^{t}}
$$

This formula expresses the probability that a voltage sag event can be recorded in the monitoring point configuration plan $X$ when a t-type fault occurs at the fault point $i$. If $\beta$ is used to represent the threshold of the observable rate of voltage sag, in order to ensure that the observable rate of voltage sag is greater than $\beta$ when various short-circuit faults occur anywhere in the whole network, the inequality constraints that should be satisfied as follows,

$$
P_{X, i}^{t} \geqslant \beta \forall i=1,2, \cdots, n \forall t=1,2,3,4
$$

In addition, the importance index of measuring point is defined. From the voltage sag of the whole network caused by short-circuit fault, if a short-circuit fault occurs at a node, which makes the average voltage sag level of the whole network lower, the node shall be equipped with power quality detector first. Thus, we define the importance matrix of measuring points MIM as follows,

$$
\begin{gathered}
S F V=\sum_{t=1}^{4} \sum_{p=\mathrm{a}}^{c} F V_{t}^{p} \\
\operatorname{MIM}(i)=\sum_{j=1}^{n} \operatorname{SFV}(i, j)
\end{gathered}
$$

Considering the economics of investment and operating costs, this paper proposes a new objective function.

$$
\min \frac{\sum X(i) \cdot\left(\frac{1}{s(i)}+\frac{1}{t(i)}\right) \cdot \operatorname{MIM}(i)}{n+1-\sum X}
$$

where, $s(i)$ and $t(i)$ are the voltage level and rated capacity of the node respectively. The numerator is equivalent to finding the minimum number of monitoring points configured considering the importance of monitoring points. The expression in the denominator is helpful to find the minimum number of monitoring points.

Taking (13) and (16) as constraints and (19) as the optimization goal, construct a single-objective optimization function. The decision vector can be solved by $0-1$ programming, binary genetic algorithm or binary particle swarm algorithm, etc. Thus we can obtain the configuration of the monitoring point.

\section{Voltage Sag Source Location State Estimation Model}

The node fault voltage is related to the three factors of fault location, fault type and fault resistance. Therefore, this paper first identifies the short-circuit fault type based on the method in literature [27], then constructs the voltage sag source location model and solves the three parameters of fault line, fault point location and fault resistance.

\section{A. Fault Location Model}

Given the network topology, branch parameters and measurement system, the line number $k$, fault distance $l$ and fault 
resistance $r_{\mathrm{f}}$ are used as optimization variables to establish the sag source location model.

The objective function of the state estimation model is as follows,

$$
J(x)=\left(Z-V\left(k, l, r_{\mathrm{f}}\right)\right)^{\mathrm{T}} W\left(Z-V\left(k, l, r_{\mathrm{f}}\right)\right)
$$

where $Z$ is the measurement vector, $Z=\left[V_{\mathrm{mla}}, V_{\mathrm{mlb}}, \cdots, V_{\mathrm{mmc}}\right]$.

Element in $Z$ represents the voltage amplitude of each phase of the monitoring points recorded by the voltage sag monitoring device; $V\left(k, l, r_{\mathrm{f}}\right)$ is the analytical expression of the voltage amplitude of the measuring point, the fault distance and the fault resistance as shown in (3)-(6). In the case of a symmetrical fault, since the three-phase voltages of the monitoring points are equal, in order to ensure the measurement redundancy, it is necessary to ensure that at least three monitoring points are simultaneously monitored when the fault occurs, that is, $b_{j}=3$ in (13).

The model contains the following implicit constraints. The fault distance $l$ needs to be limited to [0,1]. Suppose the fault resistance obeys the normal distribution and meets the $3 \sigma$ criterion in the interval $(0,16) \Omega$. In practical engineering applications, the random resistance of the fault resistance can be set according to the actual situation. Assume that there are $L$ number of transmission lines in the entire network. Then the constraint condition number of transmission of the sag source location model is shown as follows,

$$
\begin{gathered}
1 \leqslant k \leqslant L, k \in Z \\
0 \leqslant l \leqslant 1 \\
0 \leqslant r_{\mathrm{f}} \leqslant 16
\end{gathered}
$$

\section{B. Adaptive Cuckoo Algorithm}

In this paper, an adaptive cuckoo search algorithm is used to solve the above optimization model. The search path and update location formula are as follows,

$$
\begin{gathered}
x_{i}^{t+1}=x_{i}^{t}+a \otimes L(s, \lambda), i=1,2, \cdots, n \\
L(s, \lambda)=\frac{\lambda \Gamma(\lambda) \sin (\pi \lambda / 2)}{\pi}\left(\frac{1}{s^{1+\lambda}}\right),(s \geqslant 0)
\end{gathered}
$$

Where $n$ is the number of iterations, $x_{i}^{t+1}$ and $x_{i}^{t}$ are the position of generation $t$ and generation $t+1$ respectively; $a$ is the step-length control factor; $\otimes$ corresponds to point multiplication; $L(s, \lambda)$ is the Lévy random search path.

After the location is updated, compare the random number rand $\in[0,1]$ with the probability of discovery $P a$. If rand $>$ $P a$, then $x_{i}^{t+1}$ update the preference random walk method. The preference random walk formula is as follows,

$$
X_{i}^{t+1}=X_{i}^{t}+r\left(X_{j}^{t}-X_{k}^{t}\right)
$$

Where $r$ is the uniform distribution of the scaling factor in $(0,1), x_{j}^{t}$ and $x_{j}^{t}$ represents the two random solutions of the generation $t$. In order to enhance the global random search

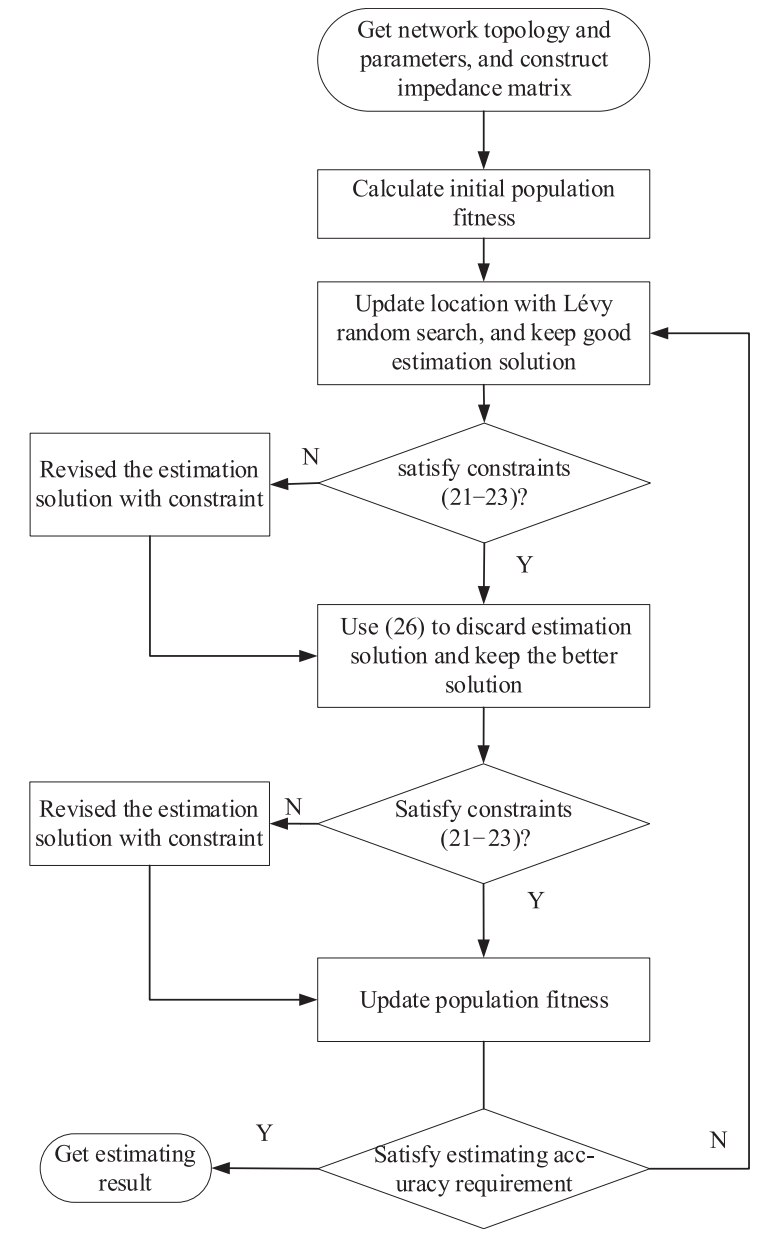

Fig. 2. Flow chart of sag source state estimation with adaptive cuckoo algorithm.

diversity and local search capabilities of the algorithm, the step length parameter $\beta$ and discovery probability $P a$ of the search path are changed from fixed values to the following update,

$$
\begin{aligned}
\beta= & \min (\operatorname{rand} n(0.8,0.3)+0.3 \\
& \left.+\left(k / k_{\max }\right), 1.8+\operatorname{rand} n(0,0.05)\right) \\
& P a=1.1-\exp \left(-\left(k / k_{\max }\right)^{0.6}\right)
\end{aligned}
$$

where $k, k_{\max }$ are the current number of iterations and the maximum number of iterations.

The flow chart of the adaptive cuckoo algorithm is shown in Fig. 2 for sag source state estimation.

\section{Sag Source Location Process}

To sum up, the flow of voltage sag source location method based on optimal configuration of monitoring points and fault parameter estimation is shown in Fig. 3. Firstly, the optimal configuration model of monitoring points considering the importance of fault resistance and measuring points is established, and the configuration scheme of monitoring points in the whole network is obtained. On this basis, the short-circuit fault type is identified, and finally the sag source location model 


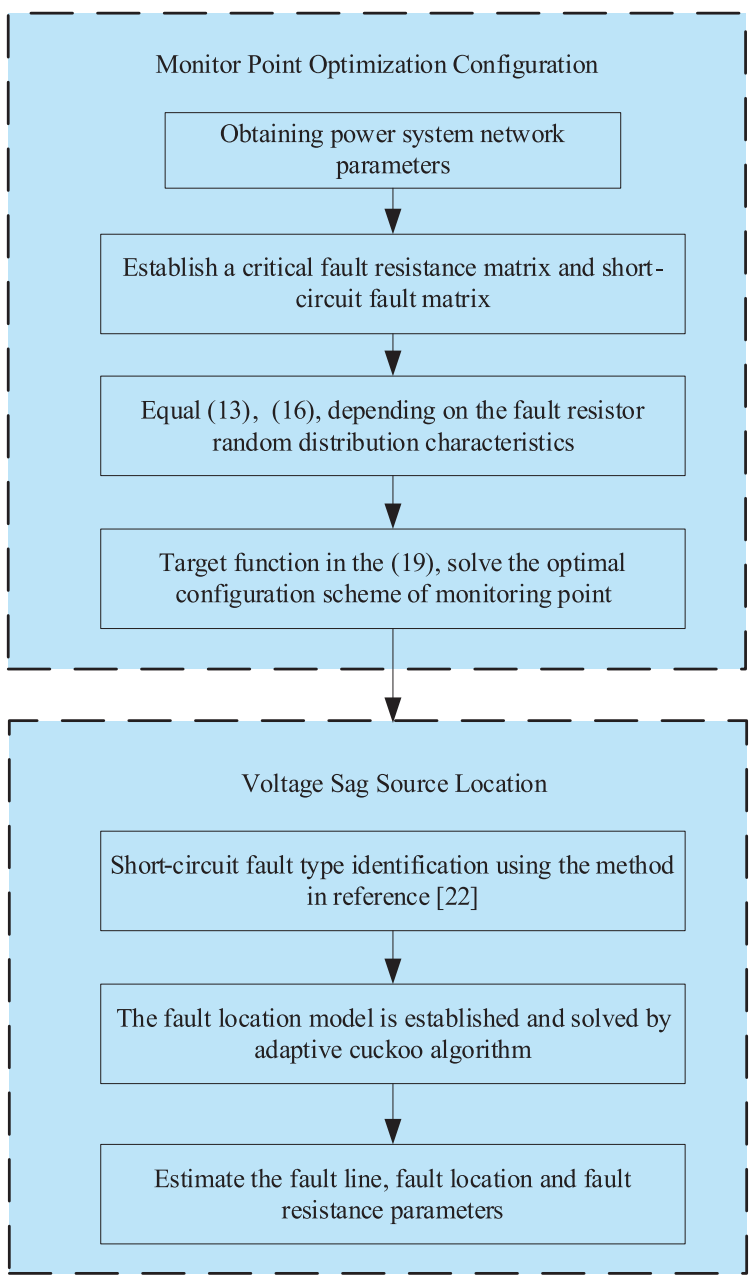

Fig. 3. Flow chart of voltage sag source location based on optimal configuration of monitoring points and fault parameter estimation.

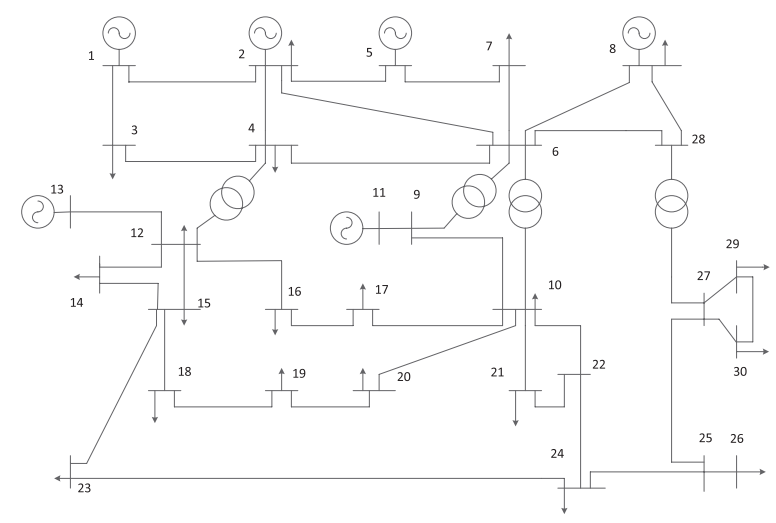

Fig. 4. IEEE-30 node system.

is solved to estimate the specific information of the fault.

\section{SimULATion AND Result}

Take the IEEE-30 node system shown in Fig. 4 as an example for simulation analysis, the detailed parameters of the system are in literature [28]. The power grid has two voltage levels of $132 \mathrm{kV}$
TABLE I

Optimal Configuration of Monitoring Points Considering the Minimum NUMBER OF OBSERVATIONS

\begin{tabular}{lccc}
\hline \hline Stage & $b_{j}$ & $\begin{array}{c}\text { Monitoring point } \\
\text { location }\end{array}$ & $\begin{array}{c}\text { Observable } \\
\text { rate }\end{array}$ \\
\hline 1 & 1 & 4,25 & 0.1483 \\
2 & 2 & $4,6,24,29$ & 0.3133 \\
3 & 3 & $2,5,7,24,25,27$ & 0.5171 \\
\hline \hline
\end{tabular}

TABLE II

Configuration Schemes of Monitoring Points Corresponding to Different Observable Rates

\begin{tabular}{lcccc}
\hline \hline Scheme & $b_{j}$ & $\beta$ & Monitoring point location & $\begin{array}{c}\text { Number of } \\
\text { monitoring } \\
\text { points }\end{array}$ \\
\hline 1 & & 0.4 & $2,4,6,24,27,30$ & 6 \\
2 & & 0.5 & $2,5,7,24,25,27$ & 6 \\
3 & & 0.6 & $2,4,7,21,26,27,29$ & 7 \\
4 & 3 & 0.7 & $3,4,7,17,24,25,30$ & 7 \\
5 & & 0.8 & $3,5,6,22,24,25,29$ & 7 \\
6 & & 0.9 & $2,5,6,10,17,24,26,27$ & 8 \\
\hline \hline
\end{tabular}

and $33 \mathrm{kV}, 6$ generators, 4 transformers, and 37 lines.

Use Matpower simulation tool to calculate the initial power flow of the system. Assuming that various types of faults occur in the busbars and lines, in order to ensure the accuracy of the optimal configuration, 9 fault points are equally spaced between the lines. The voltage sag threshold of the monitoring device is set to $0.9 \mathrm{pu}$.

\section{A. Practical Analysis of Monitoring Point Configuration}

The configuration schemes of monitoring points under different constraint conditions are shown in the Table I.

According to Table I, if the fault point is captured by at least $b_{j}$ monitoring devices, the larger the value of $b_{j}$, the more monitoring devices need to be configured, and the higher the observable rate of the minimum voltage sag at the corresponding fault point. Because the fault location estimation model in this paper needs to consider a certain degree of measurement redundancy, it is necessary to ensure that at least three monitoring points are simultaneously captured when a fault occurs at any location.

From Table II, it can be seen that the larger the value $\beta$, the higher observable rate of the corresponding voltage sag, and the corresponding number of monitoring points will increase. It can be seen from Table III that when different types of faults occur anywhere in the entire network, the probability that the monitoring system captures the voltage sag event is greater than the set $\beta$. In actual engineering applications, economic cost restrictions and sag appreciable rate requirements can be combined to build an economical and practical voltage sag monitoring system with given $\beta$.

In Table III, LG, LL, LLG, and LLL respectively indicate single-phase ground fault, two-phase short-circuit fault, twophase ground fault, and three-phase ground fault. 
TABLE III

Information on the Smallest Fault Point With Respectable Voltage Sag Rate

\begin{tabular}{lcccc}
\hline \hline Scheme & \multicolumn{4}{c}{ Fault information and sag observable rate } \\
\cline { 2 - 5 } & $\begin{array}{c}\text { Line } \\
\text { section }\end{array}$ & $\begin{array}{c}\text { Fault } \\
\text { location }\end{array}$ & Fault type & $\begin{array}{c}\text { Observable rate } \\
\text { of voltage sag }\end{array}$ \\
\hline 1 & $5-7$ & 0.2 & LLG & 0.4791 \\
2 & $9-11$ & 1 & LG & 0.5171 \\
3 & $12-13$ & 0.9 & LLG & 0.6276 \\
4 & $9-11$ & 0.5 & LLG & 0.7636 \\
5 & $9-11$ & 1 & LLLG & 0.8826 \\
6 & $12-13$ & 1 & LLLG & 0.9188 \\
\hline
\end{tabular}

TABLE IV

Fault Location Estimation Results Under DifFerent MEAsurement Errors

\begin{tabular}{lccccc}
\hline \hline $\begin{array}{l}\text { Measurement } \\
\text { error }\end{array}$ & $\begin{array}{c}\text { Line } \\
\text { section }\end{array}$ & $\begin{array}{c}\text { Fault } \\
\text { location(\%) }\end{array}$ & $\begin{array}{c}\text { Fault } \\
\text { resistance/ } \Omega\end{array}$ & $\begin{array}{c}\text { Fault } \\
\text { distance } \\
\text { error(/\%) }\end{array}$ & $\begin{array}{c}\text { Fault } \\
\text { distance } \\
\text { error } \\
(/ \%)\end{array}$ \\
\hline 0 & $(15-18)$ & 0.7498 & 4.0043 & 0.03 & 0.1 \\
$0.2 \%$ & $(15-18)$ & 0.7700 & 3.7892 & 2.67 & 5.3 \\
$0.5 \%$ & $(15-18)$ & 0.7931 & 3.5645 & 5.74 & 10.9 \\
$1 \%$ & $(15-18)$ & 0.8377 & 3.1688 & 11.69 & 20.8 \\
\hline \hline
\end{tabular}

TABLE V

Fault Location Estimation Results Under Different Fault Scenarios (0.5\% Measurement Error)

\begin{tabular}{|c|c|c|c|c|c|c|c|c|}
\hline \multirow[b]{2}{*}{ Line section } & \multirow[b]{2}{*}{ Fault type } & \multirow[b]{2}{*}{$\begin{array}{l}\text { Fault distance } \\
(\mathrm{pu})\end{array}$} & \multirow[b]{2}{*}{$\begin{array}{c}\text { Fault } \\
\text { resistance } / \Omega\end{array}$} & \multicolumn{3}{|c|}{ Proposed method } & \multicolumn{2}{|c|}{$\operatorname{Ref}[22]$} \\
\hline & & & & $\begin{array}{l}\text { Located line \& } \\
\text { distance (pu) }\end{array}$ & $\begin{array}{l}\text { Estimated } \\
\text { fault } \\
\text { resistance } \\
\quad / \Omega\end{array}$ & $\begin{array}{c}\text { Fault } \\
\text { distance } \\
\text { error }\end{array}$ & $\begin{array}{l}\text { Located line \& } \\
\text { distance (pu) }\end{array}$ & $\begin{array}{c}\text { Fault } \\
\text { distance } \\
\text { error }\end{array}$ \\
\hline $2-4$ & LG & 0.2 & 1.5 & $(2-4) 0.1988$ & 1.6414 & $0.6 \%$ & $(2-4) 0.1921$ & $3.95 \%$ \\
\hline $10-20$ & LG & 0.9 & 8 & $(10-20) \quad 0.8374$ & 9.3983 & $6.95 \%$ & $(10-20) \quad 0.8174$ & $9.1 \%$ \\
\hline $12-13$ & $\mathrm{LL}$ & 0.4 & 13 & $(12-13) 0.4281$ & 12.1330 & $7.0 \%$ & $(12-13) 0.4481$ & $12.0 \%$ \\
\hline $4-6$ & LL & 0.5 & 5 & $(4-6) 0.50221$ & 5.0251 & $0.4 \%$ & $(4-6) 0.4881$ & $2.3 \%$ \\
\hline $12-14$ & LLG & 1.0 & 16 & $(12-14) 0.9666$ & 15.224 & $3.4 \%$ & (12-14) 0.9453 & $5.5 \%$ \\
\hline $21-22$ & LLG & 0.6 & 4 & $(21-22) 0.5465$ & 3.9214 & $8.9 \%$ & $(21-22) 0.5548$ & $7.5 \%$ \\
\hline $15-18$ & LLL & 0.3 & 3 & $(15-18) 0.3275$ & 2.2297 & $9.1 \%$ & $(15-18) 0.3183$ & $6.1 \%$ \\
\hline $25-26$ & LLL & 0.8 & 9 & $(25-26) 0.8706$ & 8.191 & $8.8 \%$ & $(25-26) 0.9146$ & $14.3 \%$ \\
\hline
\end{tabular}

\section{B. Validation of Location Estimation Model}

\section{1) Influence of Measurement Error on Positioning Accuracy}

This paper chooses the monitoring point configuration scheme of scheme 5 to solve the sag source location estimation model. The voltage sag is set to occur at $75 \%$ of the line (1518) away from the $15 \#$ node, the fault type is a two-phase grounding fault, and the fault resistance is $4 \Omega$. Location estimation results under different measurement errors are shown as Table IV.

Fault distance error $=$

estimated fault distance-actual fault distance

$$
\text { length of fault line } \times 100 \%
$$

Fault resistance error $=$

estimated fault resistance - actual fault resistance actual fault resistance

\section{2) Influence of Different Fault Types on Fault Location}

By changing different fault lines and fault types, the fault location results of the method in this paper and the method in literature [22] are compared in Table V.

It can be found from Table $\mathrm{V}$ that for short-circuit faults with different fault types and fault resistances, the method in this paper can accurately locate the faulty line and the overall positioning error is small. Among them, when a two-phase short-circuit fault occurs at a fault distance of $0.5 \mathrm{pu}$ on the line (4-6), the positioning result is the most accurate, with an error of only $0.4 \%$. In the case of $0.5 \%$ measurement error, the maximum positioning error of the method in this paper is $9.1 \%$, while the maximum positioning error of the method in literature [22] is $14.3 \%$. The overall positioning error of the method in this paper is relatively small. However, both the method in this paper and the method in literature [22] must solve the fault location and fault resistance by the fault location state estimation equation under the premise of the known fault type, so the accuracy of the fault type judgment is very important.

\section{3) Influence of the Number of Monitoring Points on Positioning Accuracy}

The more the number of monitoring points is, the more fault location information will be. It can provide more correlation information between monitoring points, which will make the fault location results more robust to measurement errors. It is still assumed that the voltage sag occurs at $75 \%$ of the distance between the line $(15,18)$ and node 15 . The fault type is phasephase fault, the fault resistance is $4 \Omega$, and the measurement error is $0.2 \%$. 200 groups of measurement errors are randomly generated for testing. The monitoring point configuration schemes of scheme 2, scheme 5 and scheme 6 are tested respectively. The results are shown in Table VI.

It can be seen from Table VI that with increase of monitoring points, the average error of fault location distance decreases gradually, and the fault location result is slightly better than that of literature [24] under the same precision measurement error. Therefore, by increasing the monitoring device, the interference 
TABLE VI

Positioning Results Under Different Number of Monitoring Points (0.2\% Measurement ERror)

\begin{tabular}{lccc}
\hline \hline \multirow{2}{*}{$\begin{array}{l}\text { Number of } \\
\text { monitoring } \\
\text { points }\end{array}$} & \multicolumn{2}{c}{ Proposed method } & \begin{tabular}{c} 
Ref [24] \\
\cline { 2 - 4 }
\end{tabular} \\
\cline { 2 - 4 } & $\begin{array}{c}\text { Average } \\
\text { distance error } \\
(\%)\end{array}$ & $\begin{array}{c}\text { Maximum error } \\
(\%)\end{array}$ & $\begin{array}{c}\text { Average } \\
\text { distance error } \\
(\%)\end{array}$ \\
\hline 6(Option 2) & 2.56 & 9.57 & 2.67 \\
7(Option 5) & 2.13 & 7.65 & 2.34 \\
8(Option 6) & 1.75 & 6.87 & 1.83 \\
\hline \hline
\end{tabular}

performance to the measurement error can be improved to a certain extent. By setting a reasonable observability with increased monitoring point, it reduces the impact of measurement error to a certain extent, and then improves the accuracy of fault location.

\section{CONCLUSIONS}

Considering the influence of fault resistance on voltage sag amplitude, a new voltage sag source location estimation method is proposed based on optimal configuration of monitoring points and fault parameter estimation, which makes up for the shortcomings of the traditional model without considering fault resistance. Firstly, considering the importance of measuring points, the optimal configuration model is constructed. Then, based on the optimal configuration scheme of monitoring points considering fault resistance parameters, the sag source location model is established, and the adaptive cuckoo algorithm is used to solve the three optimization variables of fault line, fault distance and fault resistance. The feasibility and effectiveness of the proposed method are verified by the simulation of the IEEE-30 node system. The adaptive cuckoo algorithm can effectively solve the MINLP problem and improves the local search ability of the algorithm. Although there is still the small possibility of positioning error for the MINLP problems in some cases, the positioning performance is stable in the case of small measurement error or by multiple search. The measurement accuracy and synchronization of the monitoring device can effectively reduce the measurement error and improve the positioning accuracy. Therefore, how to further improve the accuracy of measurement data and ensure the accuracy of short-circuit fault type identification is the focus of the next step.

\section{REFERENCES}

[1] M. H. J. Bollen, "Voltage sags in three-phase systems," in IEEE Power Engineering Review, vol. 21, no. 9, pp. 8-15, Sept. 2001.

[2] X. -N. Xiao, in Power Quality Analysis and Control, China Electric Power Press, Beijing, China, 2004.

[3] H. -G. Yang, X. -Y. Xiao, and J. -G. Liu, "Issues and technology assessment on power quality," in Electric Power Automation Equipment, vol. 23, no. 12, pp. 1-4, Dec. 2003.

[4] M. McGranaghan and B. Roettger, "Economic evaluation of power quality," in IEEE Power Engineering Review, vol. 22, no. 2, pp. 8-12, Feb. 2002.

[5] H. -X. Ling, "Perfecting power quality indices and prospect," in Proceedings of the CSEE, vol. 34, no. 29, pp. 5073-5079, Aug. 2014.

[6] M. Kordestani, A. A. Safavi, and M. Saif, "Recent survey of large-scale systems: Architectures, controller strategies, and industrial applications," in IEEE Systems Journal, pp. 1-14, Jan. 2021, Early Access.

[7] M. Kordestani, M. Saif, M. E. Orchard, R. Razavi-Far, and K. Khorasani, "Failure prognosis and applications-A survey of recent literature," in IEEE Transactions on Reliability, vol. 70, no. 2, pp. 728-748, Jun. 2021.

[8] E. Espinosa-Juarez, A. Hernandez, and G. Olguin, "An approach based on analytical expressions for optimal location of voltage sags monitors," in IEEE Transactions on Power Delivery, vol. 4, no. 4, pp. 2034-2042, Oct. 2009

[9] W. Lv and L. -J. Tian, "Optimal allocation of voltage sag monitoring based on exposed area analysis," in Electric Power Automation Equipment, vol. 32, no. 6, pp. 45-50, Jun. 2012.

[10] Y. Liao, "Fault location observability analysis and optimal meter placement based on voltage measurements," in Electric Power Systems Research, vol. 79, no. 7, pp. 1062-1068, Jun. 2009.

[11] M. Avendano-Mora and J. V. Milanovic, "Monitor placement for reliable estimation of voltage sags in power networks," in IEEE Transactions on Power Delivery, vol. 27, no. 2, pp. 936-944, Apr. 2012.

[12] A. C. Parsons, W. M. Grady, E. J. Powers, and J. C. Soward, "A direction finder for power quality disturbances based upon disturbance power and energy," in IEEE Transactions on Power Delivery, vol. 15, no. 3, pp. 1081-1086, Jul. 2000.

[13] W. -T. Zhang and C. -S. Wang, "Transient disturbances location based on improved disturbance power and energy," in Automation of Electric Power Systems, vol. 31, no. 8, pp. 32-35, Apr. 2007.

[14] G. -Y. Lv, W. -M. Sun, X. -D. Wang, and Z. -H. Cheng, "Review on methods for voltage sag source location in power system," in Power System Protection and Control, vol. 38, no. 23, pp. 241-245, Dec. 2010.

[15] T. Tayjasanant, C. Li, and W. Xu, "A resistance sign-based method for voltage sag source detection," in IEEE Transactions on Power Delivery, vol. 20 , no. 4, pp. 2544-2551, Oct. 2005

[16] C. Li, T. Tayjasanant, and W. Xu, "Method for voltage sag source detection by investigating slope of the system trajectory," in IEEE Proceedings: Generation, Transmission and Distribution, vol. 150, no. 3, pp. 367-372, Feb. 2003.

[17] N. Hamzah, A. Mohamed, and A. Hussain, "A new approach to locate the voltage sag source using real current component," in Electric Power Systems Research, vol. 72, no. 2, pp. 113-123, Jun. 2004.

[18] A. K. Pradhan and A. Routray, "Applying distance relay for voltage sag source detection," in IEEE Transactions on Power Delivery, vol. 20, no. 1, pp. 529-531, Jan. 2005.

[19] G. -Y. Lv and X. -W. Jiang, "Location of voltage sag source based on semi-supervised SVM," in Power System Protection and Control, vol. 47, no.18, pp. 76-81, Sept. 2019.

[20] X. Li, Q. -W. Gong, H. Xiao, and B. -C. Zou, "Fault location for distribution network based on correlation analysis matching degree," in Automation of Electric Power Systems, vol. 36, no. 1, pp. 90-95, Jan. 2012.

[21] G. -C. Li, H. Wang, S. Gao, B. Liu, and Y. -M. Feng, "Fault location method for distribution network based on fault distance distribution function," in Electronic Design Engineering, vol. 25, no. 19, pp. 34-37, Oct. 2017

[22] Y. -T. Qiu, X. -Y. Xiao, H. Zhao, F. Lin, and L. Tang, "A placement approach of two-stage monitors making both voltage sag and fault position observable," in Power System Technology, vol. 38, no. 11, pp. 3166-3172, Nov. 2014.

[23] Y. -Y Liu, T. -X. Wang, D. -D. Feng, N. Ding, and S. -J. Zhou, "Multiple criterions based voltage sag location method," in Proceedings of the CSEE, vol. 35, no. 1, pp. 103-111, Jan. 2015.

[24] C. -X. Zhao, S. Tao, and X. -N. Xiao, "Fault location estimation based on voltage sag information of PQMS," in Power System Technology, vol. 40, no. 2, pp. 642-648, Feb. 2016.

[25] Y. -Y. Lin, Z. -G. Shao, Y. Zhang, Y. Zhang, D. -Y. Wu, "Voltage sag source location based on the correlation of multipoint positive sequence voltage and the typical pattern matching optimization," in Transactions of China Electrotechnical Society, vol. 32, no. 17, pp. 35-46, Sept. 2017.

[26] L. -P. Chen, X. -W. Du, W. Wang, K. -J. Cao, and Z. -C. Ren, "Optimal voltage sag monitors placement considering randomness of fault resistance," in Transactions of China Electrotechnical Society, vol. 31, no. 23, pp. 93-99, Dec. 2016

[27] C. -Y. Zhang, M. -M. Shi, Z. Fan, J. -Y. Zheng, and X. -D. Yuan, "Research on voltage sag event classification and short circuit type identification," in Electric Power Engineering Technology, vol. 37, no. 2, pp.102-107+113, Mar. 2018.

[28] University of Washington Electrical Engineering, 30 bus power flow test case [Online]. Available: http://www.ee.washington. edu/research/pstca/ pf30/pg_tca30bus.htm. 


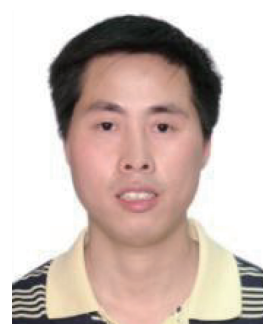

Ganyun Lv received the B.S. and M.S. degrees in control engineering from Nanjing University of Science \& Technology in 1998 and 2001, respectively, and the Ph.D. degree in electrical engineering from Shanghai Jiaotong University, China, in 2005. He is a Professor of the Electrical Engineering Department, Nanjing Institute of Technology from 2013. His research interests include power quality, model for IES, AI application in electrical power system.

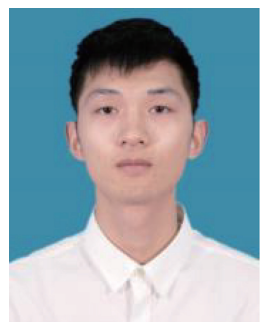

Chenjie Chu received the B.S. degree in electrical engineering from Huaiyin Institute of Technology, Huaiyin, China, in 2019, where he is currently pursuing the M.S. degree in electrical engineering.

His main research interests include power quality and AI application in electrical power system.

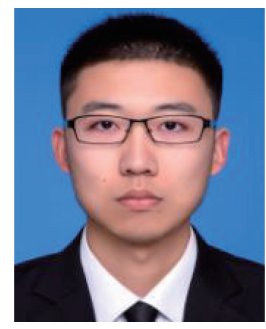

Yu Zang received the B.S. degree in electrical engineering from Xuzhou Institute of Technology, Xuzhou, China, in 2019, where he is currently pursuing the M.S. degree in electrical engineering.

His main research interests include power quality and power market.

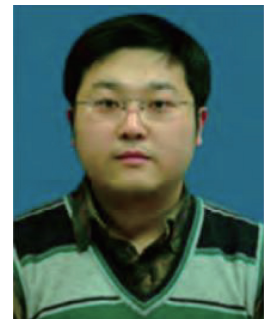

Guangyu Chen was born in Nanjing, China, in 1980, and graduated from Hohai University in 2016 with a doctor's degree in power system and its automation. He served as a Lecturer of Nanjing Institute of Technology in February 2017 and was promoted to Associate Professor in July 2020. His main research interests include power system operation and control, large power grid optimal dispatch, line loss analysis, integrated energy and electric vehicles. 